\title{
Endogenous Accounting Bias when Decision Making and Control Interact*
}

\author{
QI CHEN, Duke University
}

BRIAN MITTENDORF, Ohio State University

YUN ZHANG, George Washington University

\section{Introduction}

This paper analyzes how accounting biases endogenously arise as firm owners' optimal response to balance different stewardship uses of accounting information systems. Specifically, we identify conditions under which the nature of the stewardship use affects whether the accounting system exhibits conservative or liberal bias. ${ }^{1}$ We distinguish two types of stewardship uses of accounting information: one is to motivate the (standard) managerial efforts that affect the first moment (mean) of the firm output, and the other is to motivate the managerial efforts that facilitate the principal's decision making by affecting the second moment of the firm output (we refer to such efforts as the decision-facilitating efforts). We find that, while conservative bias may be optimal when accounting information is used only to motivate mean-increasing efforts (Kwon, Newman, and Suh 2001; Kwon 2005), liberal bias is necessary when decision making and control interact in the sense that accounting information is also used to motivate decision-facilitating efforts.

We illustrate the above idea in a simple model where an accounting system provides information relevant both for making decisions and for evaluating an agent. As a first benchmark, we demonstrate that, in the absence of evaluation considerations, an unbiased accounting (classification) system is preferred for providing useful decision-making information, thus establishing an endogenous need for an information system. As a second

* Accepted by Raffi Indjejikian. We appreciate helpful comments from Anil Arya, Shane Dikolli, Paul Fischer, Christian Hofmann, Pino Lopomo, Mohan Venkatachalam, Dae-Hee Yoon, and workshop participants at Baruch College, Emory University, Fudan University, University of Mannheim, and Tsinghua University. Financial support from Fuqua School of Business at Duke University (for Chen and Zhang), from Yale School of Management and Ohio State University Fisher College of Business (for Mittendorf), and from the George Washington University School of Business (for Yun Zhang) is gratefully acknowledged.

1. Intuitively, an accounting information system exhibits liberal (conservative) bias if it classifies a below (above) average outcome as good (bad) news. These definitions will be made precise in the model section.

Contemporary Accounting Research Vol. 27 No. 4 (Winter 2010) pp. 1-29 (C) CAAA doi:10.1111/j.1911-3846.2010.01038.x 
benchmark, we layer in a (standard) incentive problem in that the agent's effort only increases expected payoffs in the first-order stochastic dominance sense, but does not reduce the degree of uncertainty (i.e., variance) in the principal's decision-making problem. In this case, we find that the incentive problem can justify instilling a degree of conservative bias in accounting. ${ }^{2}$

As a final benchmark, we consider the case in which the agent's meanincreasing effort is observable (and contractible), and the only nontrivial moral hazard problem lies in the agent's effort to facilitate decision making by reducing uncertainty. Such a reduction in uncertainty can come from, for example, gathering more precise decision-relevant information, better diversification, and so on. For this benchmark, it is shown that bias is once again preferred in order to motivate effort, although the direction of the bias is inconsequential. That is, it is important to have a biased information system, but the bias can be either conservative or liberal.

With these benchmarks in place, we then introduce the unique aspect of our setting by considering the case in which the owner must provide incentives for the agent to exert both mean-increasing and decision-facilitating efforts. Recall, in the case of either effort problem considered independently, a weak preference arises for conservative accounting. When one considers both problems simultaneously, a stark contrast arises: the information system must exhibit liberal biases in order to motivate the agent. In fact, such a liberal bias is necessary regardless of which incentive constraints bind in equilibrium.

Intuitively, when an agent takes decision-facilitating effort to reduce uncertainty (thin the tails of a distribution), the firm seeks to reward such behavior by giving a bonus not in the event of extremely good outcomes (arising in the right tail of the distribution) but rather for an average outcome. At the same time, to motivate mean-increasing effort, the agent needs to be paid more for outcomes on the right side of the distribution than outcomes on the left side of the distribution. To manage these two incentives effectively, the firm needs an information system that classifies average outcomes as good news, that is, exhibiting modest liberal biases. ${ }^{3}$

The above result offers a new insight into the interpretation of liberal biases in firms' external financial reports. While the existence of liberal reporting biases are widely documented by empirical research (for surveys, see Healy and Wahlen 1999; Fields, Lys, and Vincent 2001), there is a lack

2. This benchmark result is generally consistent with existing literature on moral hazard and conservatism. For example, Kwon et al. (2001), Venugopalan (2001), and Kwon (2005) each present circumstances under which the desire to motivate mean-shifting efforts can give rise to conservative accounting systems under limited liability. Chen, Hemmer, and Zhang (2007) show that conservative accounting principles can help reduce firms' incentives to engage in (socially harmful) earnings management. Gigler and Hemmer (2001) and Bagnoli and Watts (2005) also consider conservatism, but in cases where both moral hazard and adverse selection are present.

3. In contrast, a conservative system classifies average outcomes as bad news. 
of consensus on their interpretation. One view is that such biases result from managers opportunistically exercising reporting discretion to advance their private benefit at owners' expense, suggesting that these biases reduce the decision-making use of accounting information for investors. An alternative view is that liberal accounting is more benign in that managers use reporting discretion to communicate their private information, suggesting that these biases increase the decision-making use of accounting information (e.g., Watts and Zimmerman 1986; Subramanyam 1996; Healy and Palepu 1996; Bowen, Rajgopal, and Venkatachalam 2010).

Our analysis suggests another view of liberal accounting bias, one rooted in the desire to effectively manage agent incentives to exert effort to improve decision making. Our explanation entails a bit of each of the above prevailing explanations: it examines a circumstance where managers' opportunistic behavior is at the forefront, but demonstrates that biases may actually reduce such opportunism and thereby improve accounting numbers' decision-making usefulness.

Comparative statics of our main result also point to some potential implications. For one, the more critical that accounting information is used for decision making in an organization, the less liberal bias one would expect to observe. We also note that, when decision-facilitating effort constitutes the pressing incentive constraint, the greater the fundamental uncertainty faced by a firm, the greater the equilibrium bias. To the extent that higher fundamental uncertainty is reflected by higher cost of capital, this result may point to a more benign explanation for the observed connection between income-increasing earnings management and higher cost of capital: higher cost of capital leads to greater bias in the reported earnings, not the other way around.

We demonstrate these primary conclusions in a stylized model to provide a parsimonious representation of endogenous biases. Despite its simplicity, the model integrates a number of general observations about the features of an accounting information system. First, we take as given that the primitive role of an accounting system is to provide information to assist decision making in uncertain environments. The decision can be either an internal decision made by the board of directors (e.g., strategic planning, mergers and acquisitions, etc.) or an external decision by outside shareholders on their optimal holding of the firm shares. Thus, we focus on a setting where the decision problem is such that more information is beneficial, thus giving endogenous demand to both an (accounting) information system and the managerial effort that reduces uncertainty.

Second, a salient feature of the accounting reporting system, as well recognized in the literature and recently summarized in Dye 2002, is essentially a process of classification: a firm is either a going concern or not, an expenditure is either a periodic expense or an investment, an asset is either short term or long term, and so on. Consequently, accounting information is often presented in highly aggregate and categorical terms. While this feature 
is a result of the specific rules, standards, and conventions that govern accounting reporting, more importantly it reflects the fact that accounting information can only help resolve some, but not all, of the firm's underlying uncertainty. To capture this feature, we follow the literature and model the accounting information system as providing a partition of the underlying state space. ${ }^{4}$ As a result, the accounting information in our model is aggregate and categorical, but is nonetheless informative about the underlying states.

Finally and perhaps most critically, we examine interactions between decision making and incentive provisions that arise naturally when an agent can take actions to facilitate decision making, in addition to the standard mean-increasing efforts. ${ }^{5}$ This is manifested in the presumption that the agent can make efforts to reduce decision uncertainty and thereby effectively improve the informativeness of the accounting system. This feature of incentive problems rooted in effort to reduce uncertainty has been previously examined in, for example, Hirshleifer and Suh 1992, Sung 1995, Meth 1996, and Demski and Dye 1999.

In Hirshleifer and Suh 1992, the phenomenon arises when a manager's efforts affect both project choice and implementation success; Sung (1995) expands the Holmstrom and Milgrom 1987 linearity result to the case in which the agent can control not only the drift (mean) of the outcome distribution but also the diffusion rate (variance); Meth (1996) examines uncertainty-reducing effort that must be motivated in order to better monitor other actions of an agent; and Demski and Dye (1999) consider contracting when an agent has private information and can take actions to control both the mean and variance of performance. In each case, the existing literature focuses on optimal contracting, not the preferred information system or bias which is the focus herein.

The question of preferred information systems has a rich history both in decision-making and control contexts. The seminal work in the former regard is Blackwell 1951, which provides necessary and sufficient conditions for information-system ranking. Taking decision control into account, Gjesdal (1982) and Grossman and Hart (1983) provide a sufficient condition for information-system ranking analogous to Blackwell ordering. Because such a ranking is only sufficient in the presence of moral hazard, Kim (1995) subsequently identified a less stringent sufficient condition rooted in likelihood ratios of different information systems. Given this existing framework, we note that the setting studied herein is one in which

4. See also Ijiri 1975, Demski 1980, Dye 1985, and Christensen and Demski 2003 for similar modeling of accounting reports as a partition of the state space.

5. In contrast, Arya, Glover, and Sivaramakrishnan (1997) consider a case in which the principal can make decisions that alter information about the agent's action. In their setting, a less informative decision-relevant signal can be useful as a substitute for a principal's commitment to a certain action so as to reduce attendant contracting costs. 
there is neither a Blackwell ordering with respect to decision making or control and neither is there an ordering with respect to Kim 1995 when one only views the usual mean-shifting agent effort. However, when the agent's efforts to reduce decision uncertainty are considered and become the binding incentive constraint, a more liberal accounting system can be shown to be preferred in accordance with Kim's 1995 ranking of information systems.

The paper proceeds as follows. Section 2 presents the basic model. Section 3 presents the results, providing three natural benchmarks and demonstrating the solution to the model and implications for endogenous liberal biases. Finally, section 4 concludes.

\section{The model}

Let $s$ denote an unobservable random variable reflecting the productive environment in which a risk-neutral firm (principal) operates. It is common knowledge that $s$ follows a uniform distribution with a mean of $k$ over the support of $[k-d, k+d], d>0$. Although $s$ can be interpreted broadly as any payoff that benefits the firm owners, to fix ideas we often interpret it as the demand for the firm's products. Before $s$ is realized, the principal can make decisions in anticipation of $s$. Examples of these actions include deciding the production capacity or building up inventories to best meet the demand. We use $i$ to denote such decisions.

In addition, for a wage payment $A$, the principal can hire an agent to directly affect the demand. We assume that the agent is risk neutral and can take two types of unobservable efforts $\left(e^{1}, e^{2}\right)$ to influence the realizations of $s .{ }^{6}$ Each effort is binary, with $e^{1} \in\left\{e_{h}^{1}, e_{l}^{1}\right\}$ and $e^{2} \in\left\{e_{h}^{2}, e_{l}^{2}\right\}$, and determines a different attribute of the distribution of $s$. Specifically, a high effort on the first action, $e_{h}^{1}$, increases the mean (i.e., the first moment, hence the superscript of 1 ) of $s$ from $k_{l}$ to $k_{h}$, with $k_{h}>k_{l}$. A high effort on the second action, $e_{h}^{2}$, reduces the variance (i.e., the second moment, hence the superscript of 2) of $s$ from $d_{l}$ to $d_{h}$, with $d_{h}<d_{l}$. Examples of the $e^{2}$ effort include conducting market research, gathering more precise decision-relevant information, reducing the inherent uncertainty of the firm's operational environment by appropriate diversification or better strategies, and so on. Effort is personally costly to the agent. We use $c^{1}$ and $c^{2}$ to denote the incremental cost of exerting a high effort on $e^{1}$ and $e^{2}$, respectively.

Given $s, i$ and $A$, the principal's net payoff is

$s-A-\alpha|i-s|$,

where $\alpha>0$ is a parameter that reflects the relative importance of the decision taken by the principal. In particular, the principal is better off if her decision $i$ matches the underlying state of the world $s$ more closely.

6. Our liberal bias results are not driven by risk neutrality. 
In the context of interpreting $s$ as demand and $i$ as capacity planning, this payoff function captures the idea that, while the firm unequivocally benefits from higher demand with a lower cost (captured by a high realized value of $s$ and a low compensation cost $A$ ), the benefits are best gleaned when the firm can set the production capacity to match closely the level of demand. While $e^{1}$ represents the standard control problem which affects only the first moment, the importance of matching decision $i$ with $s$ gives rise to the need for the agent's decision-facilitating $e^{2}$ effort. Without the need for matching, the assumption of risk neutrality implies that the optimal level for $e^{2}$ should be zero. Thus, our setting is one where the principal needs to take into account the interaction between decision making and control by motivating both efforts. Because the simultaneous consideration of both efforts constitutes the central tension in our analysis, we focus our key analysis on situations where both concerns are sufficiently severe.

We now introduce the information system. The firm can install an accounting information system that provides a partition of $s$ (reflected in a cutoff, $m$ ) prior to $i$ and $e$ 's being chosen. The system subsequently yields a (binary) public signal: if $s \geq m$ (i.e., $s$ falls in the interval $[m, k+d]$ ), the report is " $G$ " (Good); if $s<m$ (i.e., $s$ falls in the interval $[k-d, m))$, the report is " $B$ " (Bad). The binary structure reflects the aggregate and categorical nature of accounting classifications (Dye 2002) and at the same time is nonetheless informative about the underlying states.

The choice of $m$ affects the value of the accounting system to the principal in two ways. First, the principal can use the signals generated by the system to better match her decision $i$ with the environment $s$. Second, the principal can also use the signals in the incentive contract to motivate the agent to exert high efforts. Specifically, because $s$ is not observable (at least in the contracting horizon), the only publicly observable and contractible signal is the accounting classification indicating whether $s$ is Good or Bad. As a result, the agent's realized compensation can take only two values, one corresponding to the Good accounting report (denoted $A_{G}$ ) and the other to the Bad report $\left(A_{B}\right)$. Thus, in our setting, the incentive contract offered by the principal consists of three components: $\left(m, A_{G}, A_{B}\right)$. Lastly, we impose a limited liability condition on the agent: $A_{i} \geq 0, i \in(G, B)$ (i.e., the principal pays the agent, not the other way around). As will be clear soon, assuming limited liability facilitates closed-form solutions as well as ready comparison with prior literature. However, our main result does not depend on this assumption.

The principal thus solves the following maximization problem. In the program, $\operatorname{Pr}\left(j \mid e^{1}, e^{2}\right)$ reflects the probability of accounting report $j(j=G, B)$ given efforts $e^{1}$ and $e^{2} ; E\left(\cdot \mid j, e^{1}, e^{2}\right)$ represents the expectations operator conditioned on having received accounting report $j$ and agent efforts $e^{1}$ and $e^{2}$; and $c\left(e^{1}, e^{2}\right)$ reflects the agent's cost of exerting efforts $e^{1}$ and $e^{2}$. 
$\underset{\left(e^{1}, e^{2}\right),\left(m, A_{B}, A_{G}\right),\left(i_{G}, i_{B}\right)}{\max } \sum_{j=G, B} \operatorname{Pr}\left(j \mid e^{1}, e^{2}\right)\left(E\left(s-\alpha\left|i_{j}-s\right| \mid j, e^{1}, e^{2}\right)-A_{j}\right)$

subject to

$\sum_{j=G, B} A_{j} \operatorname{Pr}\left(j \mid e^{1}, e^{2}\right)-c\left(e^{1}, e^{2}\right) \geq \sum_{j=G, B} A_{j} \operatorname{Pr}\left(j \mid \tilde{e}^{1}, \tilde{e}^{2}\right)-c\left(\tilde{e}^{1}, \tilde{e}^{2}\right), \quad \forall \tilde{e}^{1}, \tilde{e}^{2}$

$A_{j} \geq 0, j=G, B$

$\sum_{j=G, B} A_{j} \operatorname{Pr}\left(j \mid e^{1}, e^{2}\right)-c\left(e^{1}, e^{2}\right) \geq \underline{U}$

\section{Program 1}

In words, Program 1 indicates that to maximize the expected payoff net of the agent's wage compensation, the principal needs to specify: (1) $\left(e^{1}, e^{2}\right)$, the desired effort combination to be implemented by the agent; (2) $\left(m, A_{G}, A_{B}\right)$, the wage payments and the installed information system; and (3) $\left(i_{G}, i_{B}\right)$, the set of decisions to be made upon observing the accounting signal. In the program, the incentive compatibility (IC) constraints guarantee that the agent finds in his own interest to choose $\left(e^{1}, e^{2}\right)$ rather than any other effort combination $\left(\tilde{e}^{1}, \tilde{e}^{2}\right)$. The wage payments need to be nonnegative due to the limited liability (LL) constraint. Finally, the individual rationality (IR) constraint guarantees that the agent receives his reservation utility $\underline{U}$. As is routine, we set $\underline{U}=0$ for simplicity; thus, the LL constraints ensure that the IR constraint is always satisfied.

Because our goal in this paper is to shed light on the optimal use of accounting bias in contracting, it is imperative to formalize a definition of bias in the information system.

Definition. Bias in accounting classification is the expected signed deviation of $\mathrm{m}$ from $\mathrm{s}$, that is, bias equals $E_{s}(s-m)$. A classification is unbiased if $E_{s}(s-m)=0$, conservative if $E_{s}(s-m)<0$, and liberal if $E_{s}(s-m)>0$.

Conceptually, the above definition captures the idea that if a below-average $s$ is classified as Good, the information system is said to exhibit liberal biases; likewise, if an above-average $s$ is classified as Bad, the information system is said to exhibit conservative biases.

The timeline of the model is summarized in Figure 1.

Although so far we have interpreted $i$ in terms of an internal decision made by firm insiders in order to fix ideas, it is by no means the only interpretation. For example, it can be a decision made by the firm's outside shareholders on whether to increase or decrease their holdings of the firm's 
Figure 1 Timeline.

\begin{tabular}{|c|c|c|c|}
\hline$t=1$ & $t=2$ & $t=3$ & $t=4$ \\
\hline $\begin{array}{l}\text { l determines } \\
\text { ion system and } \\
\text { dal payments, }\end{array}$ & $\begin{array}{l}\text { The agent either accepts or } \\
\text { rejects the contract offered, } \\
\text { and chooses }\left(e^{1}, e^{2}\right) \text {. }\end{array}$ & $\begin{array}{l}\text { Accounting signal observed. } \\
\text { The agent is compensated. } \\
\text { The principal determines } i \text {. }\end{array}$ & $\begin{array}{l}s \text { and } \\
\text { principal's } \\
\text { payoff are } \\
\text { realized. }\end{array}$ \\
\hline
\end{tabular}

shares upon the release of the firm's financial reports. Under this interpretation, the outside shareholders use the information from the firm's financial reports to gauge the firm's future performance (i.e., $s$ ) and adjust their holdings accordingly to meet their personal portfolio balancing needs. In this context, the accounting information system resembles the firm's external financial report and the key insight from our analyses applies equally to bias in both firms' internal and external information system.

\section{Results}

\section{Benchmark 1: Optimal system without moral hazard}

We start with a benchmark when there is no moral hazard consideration and the accounting information is used purely for assisting the principal's decision making (i.e., choice of $i$ ). This benchmark corresponds to the case where the firm is able to observe and directly contract on the agent's effort choices $\left(e^{1}, e^{2}\right)$ and wishes to implement high effort in each dimension. In this case, the first-best outcome can be achieved by offering a positive wage payment that covers the agent's disutility of efforts only when both $e_{h}^{1}$ and $e_{h}^{2}$ are observed. Thus, the principal's system choice amounts to finding the $m$ that helps her make the best decision $i$ to maximize her expected payoff. Lemma 1 provides the solution in this case. (For expositional ease, all proofs throughout the paper are relegated to the Appendix.)

Lemma 1. (i)For s uniformly distributed over the interval $\left[t_{1}, t_{2}\right]$, the decision $\mathrm{i}$ that maximizes the principal's utility is the median of the s-distribution (i.e., $\left(t_{1}+t_{2}\right) / 2$ ).

(ii) Without moral hazard, the optimal accounting system is unbiased, that is, $m=k_{h}$.

Lemma 1(i) shows that, given any information system $m$, the principal's optimal decision is to choose the median of the posterior distribution. In our context, this means that, if the principal observes a Good signal from the accounting information system, she would update her posterior belief about $s$ to be uniformly distributed over $s \sim U\left[m, k_{h}+d_{h}\right]$, and her optimal decision $i$ is $\frac{k_{h}+d_{h}+m}{2}$. Similarly, her optimal decision is $\frac{k_{h}-d_{h}+m}{2}$ if the signal is Bad. 
Lemma 1 (ii) shows that the best information system for the principal is an unbiased accounting system that equally partitions the state space. The intuition behind both results in Lemma 1 is due to the desire to match the decision with the state, seeking to avoid large deviations of $i$ from $s$. One may suspect that both results are driven by the assumed symmetric loss term in the principal's utility function. ${ }^{7}$ To check the robustness of Lemma 1 to an asymmetric loss function, consider the optimal decision and information system assuming the principal's net payoff is $s-A-\alpha|i-s|$ $-\beta(i-s) I(i>s)$, where $I(i>s)$ is the indicator function and $\beta \neq 0$ reflects any differential cost between overshooting and undershooting the state. (Detailed proofs are in Observation 1 of the Appendix.) In this case, while result (i) no longer holds, result (ii) regarding the optimality of the unbiased accounting system still holds. Intuitively, (ii) holds because decision $i$ is taken after observing the realized signal and can be used to deal with the asymmetric loss, whereas the cutoff choice is decided before observing any signal realization and therefore needs to minimize the ex ante odds of large deviations of the decision from the state, hence making the symmetric information system design the optimal choice.

Because this benchmark case demonstrates a preference for an unbiased system when used only for the principal's decision-making purposes, any biases that arise in equilibrium can be directly attributed to issues relating to motivating the agent to exert efforts. Further, as suggested earlier, the direction of the bias will depend on which incentive provision concerns are present. We turn to this issue next.

\section{Benchmark 2: Optimal system with moral hazard on mean-increasing effort $\left(e^{1}\right)$}

Here, we assume as before that the principal wishes to motivate high efforts on both $e^{1}$ and $e^{2}$, except that now she can observe only $e^{2}$ and must rely on incentive compensation to motivate $e^{1}$. This setting resembles the traditional modeling of incentive compensation to motivate mean-increasing effort. Given $e_{h}^{2}$ and $m$, the relevant IC constraint for the principal is:

$\operatorname{Pr}\left(G \mid e_{h}^{1}, e_{h}^{2}\right) A_{G}+\operatorname{Pr}\left(B \mid e_{h}^{1}, e_{h}^{2}\right) A_{B}-c^{1} \geq \operatorname{Pr}\left(G \mid e_{l}^{1}, e_{h}^{2}\right) A_{G}+\operatorname{Pr}\left(B \mid e_{l}^{1}, e_{h}^{2}\right) A_{B} \quad(\mathrm{IC}-1)$,

which, after substituting the probability distribution for a given $m$, can be simplified into

$A_{G}-A_{B} \geq \frac{2 d_{h} c^{1}}{k_{h}-k_{l}}$.

Together with the LL constraint, it is clear that the introduction of moral hazard on $e^{1}$ may force the principal to provide rents to the agent. Consequently, the principal must take into account such rents when deciding

7. We thank an anonymous referee for raising this concern. 
an information system. As the next proposition confirms, such a circumstance can justify a conservative accounting system.

Proposition 1. The optimal contract that motivates $\left(e_{h}^{1}, e_{h}^{2}\right)$ with moral hazard only on $e^{1}$ is:

(i) $A_{B}=0$ and $A_{G}=\left(2 d_{h} c^{1}\right) /\left(k_{h}-k_{l}\right)$ when $k_{h}<k_{l}+d_{h}$ and $A_{B}=0$ and $A_{G}=2 \mathrm{c}^{1}$ otherwise; and

(ii) $m^{*}>k_{h}$ when $k_{h}<k_{l}+d_{h}$ and $m^{*}=k_{h}$ otherwise. $^{8}$

Proposition 1(i) demonstrates the usual contracting solution with binary signals and LL: the agent is paid the minimum (zero) for a Bad signal and a bonus for a Good signal, with the bonus set high enough to create incentives for effort. Proposition 1(ii) demonstrates that when such moral hazard is nontrivially present (i.e., when $k_{h}<k_{l}+d_{h}$ ), the optimal accounting system introduces a conservative bias. When $k_{h} \geq k_{l}+d_{h}$, any $m \geq k_{l}+d_{h}$ can motivate high $e^{1}$ effort at first-best cost levels due to the moving support. The optimal cutoff is unbiased in this case due to the principal's use of accounting information for decision $i$. To restrict attention to cases in which the moral hazard problem with respect to $e^{1}$ is nontrivial, we will presume $k_{h}<k_{l}+d_{h}$ for the remainder of the analysis. As will be clear soon, our main insight is unaffected by this assumption.

At the risk of belaboring a simple point (in exchange for laying the ground for later comparison), we elaborate the intuition for Proposition 1 in a likelihood ratio framework. Specifically, given $A_{B}=0$ at the solution, the agent's incentive constraint specifies $A_{G}=\frac{c^{1}}{\operatorname{Pr}\left(G \mid e_{h}^{1}\right)-\operatorname{Pr}\left(G \mid e_{l}^{1}\right)}$. (We omit $e_{h}^{2}$ in the conditional set for notational ease.) Substituting the probability distribution for any $m$ into this expression yields the payment in part (i). Using this, the expected wage is

$\operatorname{Pr}\left(G \mid e_{h}^{1}\right) A_{G}=\frac{\operatorname{Pr}\left(G \mid e_{h}^{1}\right) c^{1}}{\operatorname{Pr}\left(G \mid e_{h}^{1}\right)-\operatorname{Pr}\left(G \mid e_{l}^{1}\right)}=\frac{c^{1}}{1-L R^{1}(m)}$

where $L R^{1}(m)=\frac{\operatorname{Pr}\left(G \mid e_{l}^{1}\right)}{\operatorname{Pr}\left(G \mid e_{h}^{1}\right)}$ is the likelihood ratio under the good signal and captures the entire effect of the information system on expected wages: the higher $L R^{1}(m)$, the higher the expected payments. Given any (interior) $m$, $L R^{1}(m)$ equals:

$L R^{1}(m)=\frac{k_{l}+d_{h}-m}{k_{h}+d_{h}-m}$

8. Our main focus is on the direction of the bias. Therefore we relegate the detailed expression for $m^{*}$ to the proofs in the Appendix for notational ease. Similarly, under the binary structure, the key component of the optimal compensation is when the agent gets a bonus payment, hence we relegate most of the expressions for the compensation payments to the Appendix as well. 
which is decreasing in $m$, reflecting that the expected wage payment is lower the more conservative the accounting system (i.e., the more stringent the requirement for recognition of good news). In fact, when only considering the moral hazard problem, the principal can achieve first-best incentive provision by setting the corner cutoff, $m \geq k_{l}+d_{h}$ (yielding $L R^{1}(m)=0$ ), to fully exploit the moving support feature of the distribution. This notion that moral hazard considerations can justify conservative biases (given LL) is similar in spirit to that in Kwon et al. 2001 and Kwon 2005.

In our setup, another consideration arises because the information system is also used for the principal's decision-making purpose. Thus the optimal cutoff weighs the desire for conservatism ( $m$ large) driven by moral hazard concerns and the desire for unbiasedness ( $m$ equal to $k_{h}$ ) dictated by decisionmaking concerns. Intuitively, the more important decision making is to the principal (the greater $\alpha$ ), the less conservative the information system.

\section{Benchmark 3: Optimal system with moral hazard on effort $e^{2}$}

Now we consider the case where the principal can observe only meanincreasing effort $e^{1}$ and must rely on incentive compensation to motivate decision-facilitating effort $e^{2}$. The relevant incentive compatibility constraint is:

$\operatorname{Pr}\left(G \mid e_{h}^{1}, e_{h}^{2}\right) A_{G}+\operatorname{Pr}\left(B \mid e_{h}^{1}, e_{h}^{2}\right) A_{B}-c^{2} \geq \operatorname{Pr}\left(G \mid e_{h}^{1}, e_{l}^{2}\right) A_{G}+\operatorname{Pr}\left(B \mid e_{h}^{1}, e_{l}^{2}\right) A_{B}(\mathrm{IC}-2)$,

which guarantees the agent (weakly) prefers $e_{h}^{2}$ to $e_{l}^{2}$. An immediate implication of (IC-2) is that it is impossible to motivate $e_{h}^{2}$ under an unbiased information system (i.e., $m=k_{h}$ ). This is because, with an unbiased system, the probability of obtaining a $G$ report (on which the compensation is based) is always one-half regardless of the effort level on $e^{2}$. Thus, a biased information system is necessary to induce $e_{h}^{2}$. The following proposition shows that in this case either a conservative or a liberal bias can help induce $e_{h}^{2}$.

Proposition 2. The optimal contract that motivates $\left(e_{h}^{1}, e_{h}^{2}\right)$ with moral hazard only on $e^{2}$ entails either of the following two solutions which generate the same (optimal) payoff to the principal: Solution 1: $m^{*}<k_{h}, A_{G}^{*}>A_{B}^{*}=0$, or Solution 2: $m^{*}>k_{h}, A_{B}^{*}>A_{G}^{*}=0$.

Under solution 1, the accounting system is liberally biased and the agent gets a reward upon a Good signal realization. This is because the liberal system leads to a higher likelihood of a realized Good signal when the agent works than when he shirks. ${ }^{9}$ Under solution 2, the information system exhibits conservative biases and the agent is paid more upon a Bad signal. This is because the conservative system leads to higher likelihood of a realized Bad accounting signal when the agent works than when he shirks. The common intuition behind both solutions is that, when the agent chooses

9. We postpone a detailed discussion of likelihood ratios until the next subsection. 
high $e^{2}$, the signal realizations are more likely to be close to the center of the distribution. As a result, the agent should be rewarded for realizations closer to the center. Proposition 2 shows that, when properly combined, a liberal system with a reward for a good outcome achieves the same payoff to the principal as a conservative system with a reward for a bad outcome.

\section{Solution to main setup with moral hazard on both $e^{1}$ and $e^{2}$}

\section{Equilibrium liberal bias}

We now turn to our main setup, assuming that the principal likes to motivate high effort on both effort dimensions. In this case, in addition to (IC-1) and (IC-2), the principal also faces one additional IC constraint:

$$
\begin{aligned}
\operatorname{Pr}\left(G \mid e_{h}^{1}, e_{h}^{2}\right) A_{G}+\operatorname{Pr}\left(B \mid e_{h}^{1}, e_{h}^{2}\right) A_{B}-c^{1}-c^{2} \geq & \operatorname{Pr}\left(G \mid e_{l}^{1}, e_{l}^{2}\right) A_{G} \\
& +\operatorname{Pr}\left(B \mid e_{l}^{1}, e_{l}^{2}\right) A_{B}
\end{aligned}
$$

Here, (IC-3) incentivizes the agent to choose $\left(e_{h}^{1}, e_{h}^{2}\right)$ over $\left(e_{l}^{1}, e_{l}^{2}\right)$.

So far we have shown that liberal bias can never be optimal to motivate $e^{1}$ while both liberal and conservative bias can be optimal for motivating $e^{2}$. Thus, at first glance, one may naturally conjecture that when both $e^{1}$ and $e^{2}$ need to be motivated the optimal system is simply conservative. As Proposition 3 shows next, this conjecture turns out to be incorrect. In order to motivate high effort on both actions, the information system is necessarily a liberal one, regardless of which incentive constraints are binding.

Proposition 3. In order to motivate $\left(e_{h}^{1}, e_{h}^{2}\right)$, the optimal information system must have $m^{*}<k_{h}$.

To see the intuition behind Proposition 3, consider how different efforts affect the likelihood of a bonus payment. Recall that, with only $e^{1}$ considered, the conservative accounting system ensured that the reward for high effort was paid only for realizations in the right tail of the $s$ distribution. This is natural if the effort in question can shift the mean of the distribution to the right. When $e^{2}$ is considered, however, paying a bonus in the tails of the distribution only dampens incentives to reduce uncertainty (i.e., thin the tails). To overcome this concern, the reward structure must pay the bonus for realizations in the center of distribution. A liberal accounting system provides such an opportunity. With a liberal system, average news is classified as Good news. Thus, if the agent can take effort to reduce uncertainty, he is more likely to get such average news and, thereby, more likely to achieve the bonus.

To elaborate, moral hazard with respect to $e^{1}$ requires payments to be increasing in the realized signal. This translates into a higher payment for a good signal than for a bad signal, that is, $A_{G}>A_{B}$ (as implied by the (IC-1) constraint). Rewriting the incentive constraint that $\left(e_{h}^{1}, e_{h}^{2}\right)$ is preferred to $\left(e_{h}^{1}, e_{l}^{2}\right)$ yields $\left[\operatorname{Pr}\left(G \mid e_{h}^{1}, e_{h}^{2}\right)-\operatorname{Pr}\left(G \mid e_{h}^{1}, e_{l}^{2}\right)\right]\left(A_{G}-A_{B}\right) \geq c^{2}$. 
A necessary condition for this to be satisfied is $\operatorname{Pr}\left(G \mid e_{h}^{1}, e_{h}^{2}\right)>\operatorname{Pr}\left(G \mid e_{h}^{1}, e_{l}^{2}\right)$ which for any $m \in\left[k_{h}-d_{h}, k_{h}+d_{h}\right]$ is equivalent to $\left(k_{h}-m\right)\left(\frac{1}{2 d_{h}}-\frac{1}{2 d_{l}}\right)>0$. Because $d_{l}>d_{h}$ by assumption, a necessary condition for the inequality to hold is $k_{h}-m>0$, that is, the information system exhibits liberal bias. Note that this requirement occurs regardless of the magnitude of the incentive problem on $e^{2}$. In fact, even in the case of $c^{2}=0$, the standard conclusion that moral hazard on mean-shifting effort favors conservative accounting systems is disabled. That is, even if effort to reduce uncertainty is costless from the agent's standpoint, it nonetheless precludes a conservative system due to a need to eliminate the temptation to increase uncertainty. In other words, even with costless variance-shifting effort, a latent incentive problem arises that necessitates a reexamination of optimal bias.

More generally, the notable aspect of our results is how they contrast starkly with the three benchmark cases. These benchmark cases show that (i) decision making alone favors an unbiased system; (ii) mean-shifting effort as the sole moral hazard problem may favor a conservative system (assuming $k_{h}-k_{l}<d_{h}$ ); and (iii) decision-facilitating effort as the sole moral hazard problem favors bias, but the direction of such bias is inconsequential and thus need not be liberal. Thus, when viewing each of the three problems independently there is no apparent demand for liberal bias. However, our results in this section stress that the simultaneous presence of moral hazard on mean-shifting and decision-facilitating efforts requires liberal bias, regardless of what constraints bind. That is, even though the incentive constraint on mean-increasing effort $e^{1}$ (IC-1) may not bind at the optimal solution, it determines the direction of the optimal bias. ${ }^{10}$ Furthermore, our liberal bias result does not require the LL assumption; liberal bias is necessary as long as incentives are needed to motivate both $e^{1}$ and $e^{2}$.

\section{The optimal extent of liberal bias}

A question that naturally follows is how liberal the accounting system will be in equilibrium. To address this most succinctly, consider the scenario in which the effort of primary interest, $e^{2}$, comprises the pressing incentive problem. In this case, the relevant (binding) incentive constraint is (IC-2) which requires that the agent prefers $\left(e_{h}^{1}, e_{h}^{2}\right)$ to $\left(e_{h}^{1}, e_{l}^{2}\right)$. Such a scenario is ensured so long as the second-moment moral hazard problem is sufficiently pronounced or $c^{2}$ is big enough. While this circumstance highlights the primary forces of interest most succinctly, we note that similar results are obtained when $c^{2}$ is small and different constraints bind. ${ }^{11}$ With $A_{B}=0$ at the optimal, (IC-2) can be simplified as

10. In other words, the principal's problem here when only (IC-2) binds is not the same as the principal's problem in benchmark 2 when the mean-increasing effort $\left(e^{1}\right)$ is observable.

11. For completeness, the Appendix presents the (rather unwieldy) complete characterization, and interested readers are encouraged to contact the authors for the full details. 
$A_{G}=c^{2} /\left[\operatorname{Pr}\left(G \mid e_{h}^{1}, e_{h}^{2}\right)-\operatorname{Pr}\left(G \mid e_{h}^{1}, e_{l}^{2}\right)\right]$.

Employing the relevant probability distributions for any interior $m$, this entails

$A_{G}=2 d_{h} d_{l} c^{2} /\left[\left(d_{l}-d_{h}\right)\left(k_{h}-m\right)\right]$.

We now turn to the optimal information system in this case. Using the above payment, the expected wage is

$\operatorname{Pr}\left(G \mid e_{h}^{1}, e_{h}^{2}\right) A_{G}=\frac{c^{2}}{\left[1-\operatorname{Pr}\left(G \mid e_{h}^{1}, e_{l}^{2}\right) / \operatorname{Pr}\left(G \mid e_{h}^{1}, e_{h}^{2}\right)\right]}=\frac{c^{2}}{1-L R^{2}(m)}$,

where $L R^{2}(m)=\operatorname{Pr}\left(G \mid e_{h}^{1}, e_{l}^{2}\right) / \operatorname{Pr}\left(G \mid e_{h}^{1}, e_{h}^{2}\right)$. As before, the information system's effect on expected wages is captured entirely by the likelihood ratio under the Good signal: the higher $L R^{2}(m)$, the higher the expected payments. Given any (interior) cutoff $m<k_{h}, L R^{2}(m)$ equals:

$L R^{2}(m)=\frac{d_{h}\left(k_{h}+d_{l}-m\right)}{d_{l}\left(k_{h}+d_{h}-m\right)}$

Equation 2 shows that $L R^{2}(m)$ increases in $m$, reflecting that the expected wage payment is lower the more liberal the accounting system (i.e., the less stringent the requirement for recognition of Good news). So, not only is a liberal accounting system necessary to motivate effort, the incentive problem viewed alone would suggest the more liberal the accounting system the better.

More generally, one can link this result that a moral hazard problem for effort that reduces decision uncertainty leads to a preference for liberal accounting to Kim's 1995 ranking of information systems. In particular, as detailed in the Appendix, the Likelihood Ratio Distribution Function in this case, $L_{m}^{2}(z)$, is:

$$
\begin{array}{ll}
L_{m}^{2}(z)=0 & \text { for } z<1-\frac{d_{h}\left(m-k_{h}+d_{l}\right)}{d_{l}\left(m-k_{h}+d_{h}\right)} ; \\
L_{m}^{2}(z)=\frac{m-\left(k_{h}-d_{h}\right)}{2 d_{h}} & \text { for } 1-\frac{d_{h}\left(m-k_{h}+d_{l}\right)}{d_{l}\left(m-k_{h}+d_{h}\right)} \leq z<1-\frac{d_{h}\left(k_{h}+d_{l}-m\right)}{d_{l}\left(k_{h}+d_{h}-m\right)} \\
L_{m}^{2}(z)=1 & \text { for } z \geq 1-\frac{d_{h}\left(k_{h}+d_{l}-m\right)}{d_{l}\left(k_{h}+d_{h}-m\right)}
\end{array}
$$

Using (3), straightforward algebra confirms that, for any $m^{\prime}<m^{\prime \prime}<k_{h}$ (the latter inequality assured from Proposition 3$), L_{m^{\prime}}^{2}(z)$ represents a mean preserving spread of $L_{m^{\prime \prime}}^{2}(z)$, thereby confirming that Kim's 1995 condition supports the preference for a more liberal system when the moral hazard with respect to $e^{2}$ is the pressing concern. By employing the sufficient condition for information-system ranking in Kim 1995, this derivation confirms that such a preference would persist even under risk aversion provided that the 
incentive constraint is binding in equilibrium, thereby confirming the basic conclusion herein is not sensitive to the presumption of LL. ${ }^{12}$

However, because the information system is not used exclusively for incentives but must also provide information directly for decision-making purposes, an interior solution is obtained that balances the desire for unbiasedness (stipulated by decision making) and the desire for liberal bias (stipulated by incentive provisions).

Proposition 4. There exist $\underline{c}^{2}$ and $\underline{\alpha}$ such that when $c^{2} \geq \underline{c}^{2}$ and $\alpha \geq \underline{\alpha}$, the optimal contract that motivates $\left(e_{h}^{1}, e_{h}^{2}\right)$ features $A_{G}^{*}>A_{B}^{*}=0$ and $m^{*}<k_{h}$. Furthermore, in this case, the optimal information system cutoff, $m^{*}$, is (weakly) increasing in $\alpha$, (weakly) decreasing in $c^{2}$, (strictly) decreasing in $d$, and (weakly) increasing in $\Delta$, where $d \equiv d_{h}$ and $\Delta \equiv d_{l}-d$.

Intuitively, Proposition 4 performs comparative static analyses when the (IC-2) constraint is the pressing (binding) constraint which is guaranteed by the two (lower) bounds $\underline{c}^{2}$ and $\underline{\alpha}$. Specifically, it shows that (i) the greater the relative importance of decision making (as captured by a larger $\alpha$ ), the closer the information system is to the unbiased benchmark and (ii) the more pronounced the incentive problem (as captured by a larger $c^{2}$ ), the more the firm relies on liberal bias to alleviate it. In short, the proposition suggests that the more important incentive provisions are for an organization, the more bias one would expect in equilibrium. Importantly, such bias arises not due to manipulation by the agent or even appeasement by the principal, but rather as an optimal means of providing incentives. Though the natural temptation when observing such biases in practice (e.g., upward earnings management) is to presume unchecked managerial opportunism, this result clearly points to an alternative.

Interestingly, two additional comparative statics can be gleaned from Proposition 4 by defining $d \equiv d_{h}$ and $\Delta \equiv d_{l}-d$. Clearly, with this reparameterization, the optimal information system cutoff $m$ is a decreasing function of $d$ and an increasing function of $\Delta$. Or equivalently, the equilibrium liberal bias is increasing (decreasing) in $d(\Delta)$. Intuitively, as $d(\Delta)$ increases (decreases), for the same cutoff, the likelihood of obtaining the $G$ signal whether working or shirking on $e^{2}$ becomes increasingly harder to distinguish. Thus, the principal needs to bias the system even more in order to restore the agent's incentives to exert the second-moment effort. In this case, if $d$ is interpreted as the fundamental uncertainty of a firm in equilibrium and roughly proxied by cost of capital, there is strong empirical

12. Interestingly, we note that Kim's 1995 condition does not allow a succinct comparison of information systems when only moral hazard on $e^{1}$ is present (the case in section 3 ). Thus, as noted in Kwon et al. 2001, the conclusion that such incentive considerations warrant conservative accounting is more context dependent and depends crucially on the assumption of the LL constraint. 
evidence consistent with the result presented here (see, e.g., Francis, LaFond, Olsson, and Schipper 2005). While such a connection has been viewed as a sign that earnings bias is harmful (and thereby increases cost of capital), our results suggest that the relationship could be the reverse: the greater the fundamental uncertainty (cost of capital), the greater the need for a liberally biased accounting system.

We next explore a few robustness checks to discuss the extent to which the primary conclusions herein persist in other settings. First, in the current model, the costs of effort are assumed to be additive, thus excluding the possibility of either increasing or decreasing marginal cost of efforts. Suppose now we consider a general cost function $c\left(e^{1}, e^{2}\right)$, where $e^{1} \in\left\{e_{h}^{1}, e_{l}^{1}\right\}$, $e^{2} \in\left\{e_{h}^{2}, e_{l}^{2}\right\} .{ }^{13}$ Without loss of generality, we can normalize $c\left(e_{l}^{1}, e_{l}^{2}\right)$ to 0 and re-parameterize the problem by defining $c^{1}, c^{2}$, and $c^{3}$ such that $c\left(e_{h}^{1}, e_{h}^{2}\right)-c\left(e_{l}^{1}, e_{h}^{2}\right)=c^{1}, c\left(e_{h}^{1}, e_{h}^{2}\right)-c\left(e_{h}^{1}, e_{l}^{2}\right)=c^{2}$, and $c\left(e_{h}^{1}, e_{h}^{2}\right)=c^{1}+c^{2}+c^{3}$. With this change of variables, (IC-1) and (IC-2) are not affected, while (IC-3) becomes

$$
\operatorname{Pr}\left(G \mid e_{h}^{1}, e_{h}^{2}\right) A_{G}+\operatorname{Pr}\left(B \mid e_{h}^{1}, e_{h}^{2}\right) A_{B}-c^{1}-c^{2}-c^{3} \geq \operatorname{Pr}\left(G \mid e_{l}^{1}, e_{l}^{2}\right) A_{G}+\operatorname{Pr}\left(B \mid e_{l}^{1}, e_{l}^{2}\right) A_{B},
$$

with an extra term $c^{3}$ appearing on the left-hand side which can be loosely interpreted as incremental cost of exerting $\left(e_{h}^{1}, e_{h}^{2}\right)$ relative to only working hard on one effort. It is easy to see that introducing $c^{3}$ will not have any effect on Proposition 1 (which has only the (IC-1) constraint), Proposition 2 (which has only the (IC-2) constraint), or Proposition 3 (which is driven by (IC-1) and (IC-2)). In other words, the key insight of the paper that liberal accounting bias emerges when both types of efforts need to be motivated is unaffected by the addition of $c^{3}$. In addition, $c^{3}$ (provided it is not too negative) would only change the bound $\underline{c}^{2}$ in Proposition 4, which could be increasing in $c^{3}$.

As a second robustness check, if we allow the possibility that meanshifting efforts also increase variance, it is readily confirmed that a liberal accounting system is still necessary to induce both $e_{h}^{1}$ and $e_{h}^{2}$ (presuming it is feasible to do so). As an example of when such a scenario could arise, it can be argued that investing in an emerging market yields a higher return but at a higher risk than investing domestically, yet there are still things that managers can do to reduce uncertainty about foreign operations such as prudently hedging against exchange rate fluctuations, cultivating relations with local governments to alleviate political risk, or finding reliable local partners.

Third, our focus is to understand the property of the information system firms use for motivating incentives. In our model, this information system is also assumed to be used by the principal for decision-making purposes. The purpose of this assumption is to operationalize the idea that

13. We thank an anonymous referee for bringing our attention to this issue. 
the second moment effort is valuable. This is not a crucial assumption. As long as both the mean-increasing and variance-reduction efforts are valuable to the firm, and as long as the control system is used to motivate both efforts, the control system necessarily entails liberal bias.

Lastly, while we focus on a binary partition of $s$ for easy comparison with existing work, clear definition of biases, and tractability, our primary conclusions are not unique to the binary case. Consider the following example: $k_{l}=36 ; k_{h}=40 ; d_{l}=18 ; d_{h}=10 ; \alpha=4 ; c^{1}=3 / 2 ; c^{2}=1 / 2 .{ }^{14}$ Say the issue were not a partition of $s$ into two regions but instead a partition into four regions. For this example, the optimal system without moral hazard is of quartiles (cutoffs of 35, 40, and 45), a result analogous to benchmark 1. Much like in the binary case, the introduction of moral hazard on $e^{1}$ (benchmark 2) yields an optimal system with uniformly more stringent cutoffs (approximately 35.3, 40.7, and 46). Also, just as in the binary case, joint consideration of moral hazard on $e^{1}$ and $e^{2}$ yields an optimal system with uniformly less stringent cutoffs than either benchmark (approximately 33.7, 39.1, and 44.6).

As a final consideration, we examine the circumstances under which motivating high effort is, in fact, optimal. Of course, if the costs of motivating effort are sufficiently small (i.e., $c^{1}$ and $c^{2}$ small), motivating effort on both dimensions is optimal. Given our main focus on the decision-facilitating effort $\left(e^{2}\right)$, it seems worthwhile to examine the circumstances under which motivating that effort is optimal and the ensuing comparative statics. Proposition 5 presents such a comparison.

Proposition 5. For $c^{1}$ sufficiently small and $\alpha \geq \underline{\alpha}$, the optimal contract motivates high effort for $e^{1}$ and there exists $\bar{c}^{2}$ such that:

(i) $\forall c^{2} \leq \bar{c}^{2}$, motivating high effort for $e^{2}$ is optimal, the preferred accounting system entails liberal bias, and the magnitude of bias is increasing in $c^{2}$; and

(ii) $\forall c^{2}>\bar{c}^{2}$, motivating low effort for $e^{2}$ is optimal, the preferred accounting system entails conservative bias, and the magnitude of bias is constant with respect to $c^{2}$.

In effect, Proposition 5 provides a holistic view of the contractual solution to the incentive problems outlined in Propositions 1 and 4 . When the principal seeks only to motivate $e^{1}$ effort (part (ii)), the optimal information system is conservative, and the cost of $e^{2}$ effort $c^{2}$ is inconsequential. However, when the principal seeks also to motivate $e^{2}$ effort, the information system is liberal to the extent dictated by $c^{2}$. Taken together, the results suggest a nonmonotonic relationship between the extent of the

14. It can be confirmed that for this example motivating effort on both dimensions is optimal for the principal. Also, to rule out a desire to burn profits, we presume monotonic payments (for more on this, see, e.g., Meth 1996). Contact the authors for full details of the example. 
Figure 2 Accounting bias as a function of $c^{2}$.

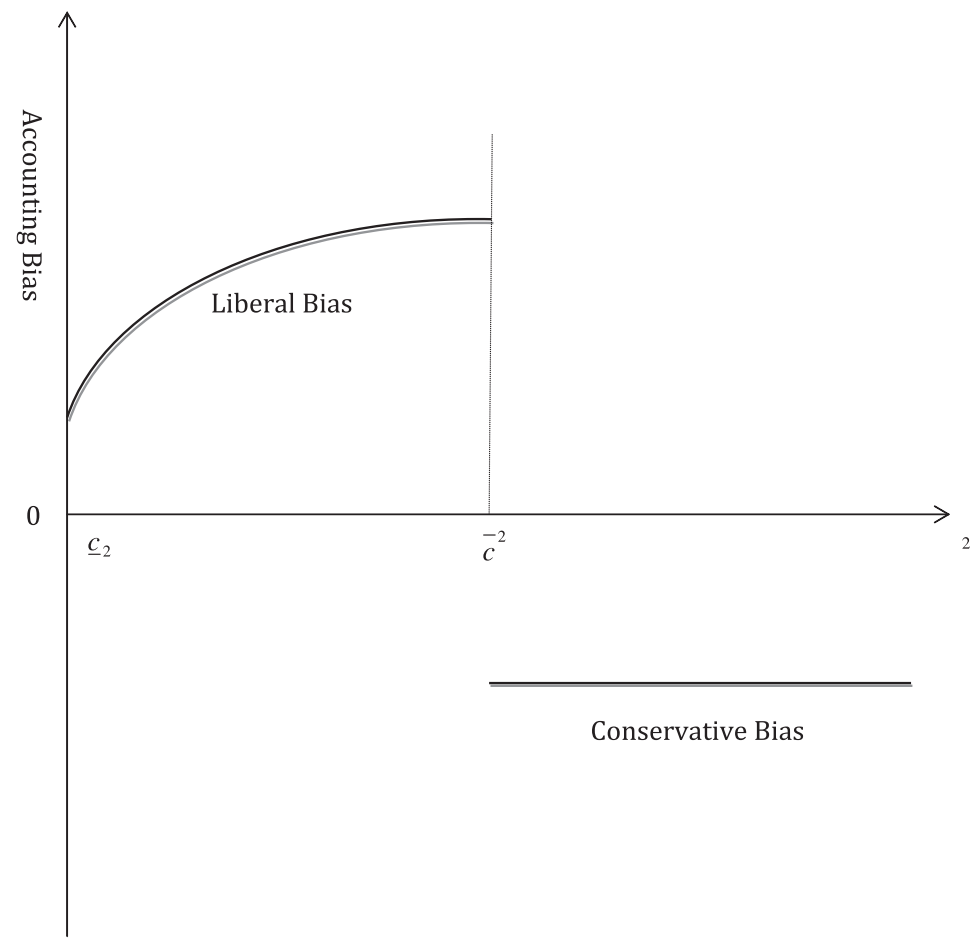

decision-facilitating incentive problem (i.e., the magnitude of $c^{2}$ ) and the extent and direction of bias in the optimal information system as depicted intuitively in Figure 2.

Our results also seem to be consistent with casual empirical observations that firms in more traditional industries (where incentive provision for traditional mean-increasing effort may be relatively more important) do seem to practice more conservative accounting while firms in high-risk, high-growth industries (where incentive provision for decision-facilitating effort may be relatively more important) seem to practice more liberal accounting.

\section{Conclusion}

This paper presents a simple model of biases in an accounting system that is employed for both decision-making and incentive provisions. We demonstrate a preference for unbiased classification for the sake of decision making and show how incentive provisions can affect both the optimality and the nature of the biases. Consistent with prior literature, we demonstrate that, when incentive provision entails efforts that are independent of the subsequent decision (i.e., they increase expected payoffs but do not affect 
subsequent decision making per se), conservatism may arise. In contrast, when incentive provision also entails efforts that can reduce decision uncertainty and thereby increase payoffs by improving decision making, a different picture emerges. In that case, the optimal accounting system exhibits liberal biases so as to reward an agent not just for abnormally high outcomes (also indicative of little effort to reduce uncertainty) but also for average outcomes (indicative of efforts to reduce uncertainty).

Besides providing a justification for liberal biases rooted in optimal incentive provisions, the results also provide a framework for when one would expect certain biases to arise. Further, by explicitly examining information systems when various incentive provision concerns are present and interact, the results highlight the important aspects of such interactions on the underlying conclusions, most notably the reversal in the preferred bias.

As a concluding provocation, we note that the results here may suggest that the (perceived) increase in aggressive accounting in recent years could be attributed to a shift toward an information economy (where effort affects decision making) rather than just an indicator of a diminished ethic among accounting practitioners.

\section{Appendix}

\section{Proof of Lemma 1}

(i) Because $s$ is uniformly distributed over the interval $\left[t_{1}, t_{2}\right]$ with $t_{2}>t_{1}$, the principal's expected gross payoff from decision $i$ that is interior to the support, that is, $i \in\left(t_{1}, t_{2}\right)$, is:

$\int_{t_{1}}^{t_{2}}[s-\alpha|i-s|] \frac{1}{t_{2}-t_{1}} d s=\frac{t_{1}+t_{2}}{2}-\alpha \frac{i^{2}-i\left(t_{1}+t_{2}\right)+\left(t_{1}^{2}+t_{2}^{2}\right) / 2}{t_{2}-t_{1}}$

Because (A1) is strictly concave in $i$, setting its derivative with respect to $i$ equal to zero yields the optimal $i=\left(t_{1}+t_{2}\right) / 2$. The principal's gross payoff is then obtained by substituting $i$ in (A1). Finally, it is straightforward to show that any non-interior choice of $i \notin\left(t_{1}, t_{2}\right)$ is strictly dominated by $i=\left(t_{1}+t_{2}\right) / 2$.

(ii) Using (i), we obtain the principal's expected payoff gross of wage payment with an interior cutoff $m \in\left(k_{h}-d_{h}, k_{h}+d_{h}\right)$ :

$$
\begin{aligned}
& k_{h}-\alpha \frac{m-\left(k_{h}-d_{h}\right)}{2 d_{h}} \int_{k_{h}-d_{h}}^{m} \frac{\left|s-\left(k_{h}-d_{h}+m\right) / 2\right|}{m-\left(k_{h}-d_{h}\right)} d s \\
& -\alpha \frac{k_{h}+d_{h}-m}{2 d_{h}} \int_{m}^{k_{h}+d_{h}} \frac{\left|s-\left(k_{h}+d_{h}+m\right) / 2\right|}{k_{h}+d_{h}-m} d s=k_{h}-\alpha \frac{\left(m-k_{h}\right)^{2}+\left(d_{h}\right)^{2}}{4 d_{h}}
\end{aligned}
$$

Because (A2) is strictly concave in $m$, setting its derivative with respect to $m$ equal to zero yields an interior optimal $m=k_{h}$, under which the optimal principal's gross payoff is $k_{h}-\alpha \frac{d_{h}}{4}$. Finally, any noninterior choice of 
$m \notin\left(k_{h}-d_{h}, k_{h}+d_{h}\right)$ yields an expected gross payoff of $k_{h}-\alpha \frac{d_{h}}{2}$ for the principal which is strictly dominated by that under $m=k_{h}$.

Q.E.D.

\section{Observation 1}

When the principal's utility is $s-\alpha|i-s|-\beta(i-s) \mathrm{I}(i>s)-A$ where $\beta \neq 0$,

(i) For $s$ uniformly distributed over the interval $\left[t_{1}, t_{2}\right]$, the optimal decision $i$ is $\frac{\alpha\left(t_{1}+t_{2}\right)+\beta t_{1}}{2 \alpha+\beta}$.

(ii) Without moral hazard, the optimal accounting system is unbiased, that is, $m=k_{h}$.

\section{Proof of Observation 1}

(i) Because $s$ is uniformly distributed over the interval $\left[t_{1}, t_{2}\right]$ with $t_{2}>t_{1}$, the principal's expected gross payoff from decision $i$ that is interior to the support, that is, $i \in\left(t_{1}, t_{2}\right)$, is:

$$
\begin{aligned}
\int_{t_{1}}^{t_{2}}[s-\alpha|i-s|-\beta(i-s) \mathrm{I}(i>s)] \frac{1}{t_{2}-t_{1}} d s & =\frac{t_{1}+t_{2}}{2} \\
& -\alpha \frac{i^{2}-i\left(t_{1}+t_{2}\right)+\left(t_{1}^{2}+t_{2}^{2}\right) / 2}{t_{2}-t_{1}} \\
& -\beta \frac{\left(i-t_{1}\right)^{2}}{2\left(t_{2}-t_{1}\right)}
\end{aligned}
$$

Because (A3) is strictly concave in $i$, setting its derivative with respect to $i$ equal to zero yields the optimal $i=\frac{\alpha\left(t_{1}+t_{2}\right)+\beta t_{1}}{2 \alpha+\beta}$. Finally, it is straightforward to show that any noninterior choice of $i \notin\left(t_{1}, t_{2}\right)$ is strictly dominated by $i=\frac{\alpha\left(t_{1}+t_{2}\right)+\beta t_{1}}{2 \alpha+\beta}$. Observe that, when $\beta>0(\beta<0)$, the optimal decision $i$ is smaller (larger) than the median $\frac{t_{2}+t_{1}}{2}$ because the principal suffers a greater loss from taking a decision that is above (below) the state of nature and hence optimally downwardly (upwardly) deviates from the median.

(ii) Using (i), we obtain the principal's expected payoff gross of wage payment with an interior cutoff $m \in\left(k_{h}-d_{h}, k_{h}+d_{h}\right)$ :

$$
\begin{array}{r}
k_{h}-\frac{1}{2 d_{h}}\left\{\frac{\alpha^{2} \beta\left(m-k_{h}+d_{h}\right)^{2}}{2(2 \alpha+\beta)^{2}}+\alpha\left\{\frac{\left[(\alpha+\beta)\left(k_{h}-d_{h}\right)+\alpha m\right]^{2}}{(2 \alpha+\beta)^{2}}\right.\right. \\
\left.-\frac{(\alpha+\beta)\left(k_{h}-d_{h}\right)+\alpha m}{(2 \alpha+\beta)}\left(k_{h}-d_{h}+m\right)+\frac{\left(k_{h}-d_{h}\right)^{2}+m^{2}}{2}\right\} \\
+\frac{\alpha^{2} \beta\left(m-k_{h}-d_{h}\right)^{2}}{2(2 \alpha+\beta)^{2}}+\alpha\left\{\frac{\left[(\alpha+\beta) m+\alpha\left(k_{h}+d_{h}\right)\right]^{2}}{(2 \alpha+\beta)^{2}}\right. \\
\left.\left.-\frac{(\alpha+\beta) m+\alpha\left(k_{h}+d_{h}\right)}{(2 \alpha+\beta)}\left(k_{h}+d_{h}+m\right)+\frac{\left(k_{h}+d_{h}\right)^{2}+m^{2}}{2}\right\}\right\} .
\end{array}
$$

Taking the derivative of the above expression with respect to $m$ and setting it to zero shows that the principal's expected gross payoff exactly is 
again minimized at $k_{h}$. The case of a noninterior cutoff can be eliminated as before.

Q.E.D.

\section{Proof of Proposition 1}

With $e^{2}$ observable and contractible, we are essentially dealing with a single-dimensional moral hazard problem. To solve the problem, consider two cases. For case 1 , say $k_{h} \geq k_{l}+d_{h}$. In this case, the first-best outcome in benchmark one can be obtained by the following contract: $m=k_{h}$, $A_{B}=0$, and $A_{G}=2 c^{1}$. Further, from Lemma $1, m=k_{h}$ is necessary to achieve this benchmark. Case $2, k_{h}<k_{l}+d_{h}$, is a bit more complicated.

We proceed with case 2 in two steps. First, we solve a restricted program in which the information system cutoff $\mathrm{m}$ is restricted to be interior, that is, $m \in\left[k_{h}-d_{h}, k_{l}+d_{h}\right]$. (Note that given $k_{h}<k_{l}+d_{h},\left[k_{h}-d_{h}, k_{l}\right.$ $\left.+d_{h}\right]$ is not an empty set.)

Second, we then show that the principal's expected payoff is higher under the optimal interior solution than when $m$ is restricted to be noninterior.

Step 1. $m$ is restricted to be interior.

Note first, by Lemma 1 the principal's optimal choice of $i$ is $i=\left(k_{h}+\right.$ $\left.d_{h}+m\right) / 2\left(i=\left(k_{h}-d_{h}+m\right) / 2\right)$ if the accounting signal is Good (Bad). In order to motivate the agent to exert $e_{h}^{1}$, the agent's IC constraint must be satisfied, that is,

$$
\begin{array}{r}
\frac{k_{h}+d_{h}-m}{2 d_{h}} A_{G}+\frac{m-\left(k_{h}-d_{h}\right)}{2 d_{h}} A_{B}-c^{1} \geq \frac{k_{l}+d_{h}-m}{2 d_{h}} A_{G}+\frac{m-\left(k_{l}-d_{h}\right)}{2 d_{h}} A_{B} \\
\Leftrightarrow A_{G}-A_{B} \geq \frac{2 d_{h} c^{1}}{k_{h}-k_{l}} .
\end{array}
$$

This implies that, in order to motivate $e_{h}^{1}$, it is necessary to offer the agent a higher wage compensation when the accounting signal is Good. Thus, substituting in the expression for $i$, the restricted program is

$$
\begin{aligned}
& \max _{A_{B}, A_{G}, m} k_{h}-\alpha \frac{\left(m-k_{h}\right)^{2}+d_{h}^{2}}{4 d_{h}}-\left[\frac{k_{h}+d_{h}-m}{2 d_{h}} A_{G}+\frac{m-\left(k_{h}-d_{h}\right)}{2 d_{h}} A_{B}\right] \\
& \text { subject to } \quad A_{G}-A_{B} \geq \frac{2 d_{h} c^{1}}{k_{h}-k_{l}} \\
& \quad m \in\left[k_{h}-d_{h}, k_{l}+d_{h}\right] .
\end{aligned}
$$

\section{Program 2}

Clearly, at the optimal solution, $A_{G}=\frac{2 d_{h} c^{1}}{k_{h}-k_{l}}>A_{B}=0$. Because the objective function is strictly concave in $m$, the first-order condition with respect to $m$, $m^{*}=k_{h}+\frac{2 d_{h} c^{1}}{\alpha\left(k_{h}-k_{l}\right)}$, is both necessary and sufficient for optimality if it is interior. Notice that $m^{*} \in\left[k_{h}-d_{h}, k_{l}+d_{h}\right]$, hence interior, if and only if $c^{1} \leq \frac{\alpha\left(d_{h}-k_{h}+k_{l}\right)\left(k_{h}-k_{l}\right)}{2 d_{h}}$. Thus, $m^{*}=k_{h}+\frac{2 d_{h} c^{1}}{\alpha\left(k_{h}-k_{l}\right)}$ is the optimal solution to Program 2 when $c^{1} \leq \frac{\alpha\left(d_{h}-k_{h}+k_{l}\right)\left(k_{h}-k_{l}\right)}{2 d_{h}}$. When $c^{1}>\frac{\alpha\left(d_{h}-k_{h}+k_{l}\right)\left(k_{h}-k_{l}\right)}{2 d_{h}}$, the optimal 
solution is on the boundary $k_{l}+d_{h}$. What's more, the optimal $m$ corresponds to a conservative accounting system as $E\left[s-m^{*} \mid\left(e_{h}^{1}, e_{h}^{2}\right)\right]=$ $\max \left\{-\frac{2 d_{h} c^{1}}{\alpha\left(k_{h}-k_{l}\right)},-\left(k_{l}+d_{h}-k_{h}\right)\right\}<0$.

Step 2. $m$ is restricted to be noninterior.

When $m$ is restricted to be noninterior, that is, $m \notin\left[k_{h}-d_{h}, k_{l}+d_{h}\right]$, it is easy to show that the principal's optimal $m$ is (infinitely close to) $k_{l}+d_{h}$ and the optimal wage compensation is $A_{G}=\frac{2 d_{h} c^{1}}{k_{h}-k_{l}}>A_{B}=0$. The idea here is that, for $m>k_{l}+d_{h}$, low effort cannot lead to a Good signal and as a result a judiciously chosen bonus can achieve the first-best outcome for providing incentives (i.e., the expected wage compensation is $c^{1}$ ). And, because decision making demands $m$ closer to $k_{h}$, the preferred $m>k_{l}+d_{h}$ is simply (infinitely close to) $k_{l}+d_{h}$. As the principal's payoff function is continuous in $m$ and the boundary solution of $m=k_{l}+d_{h}$ has been considered above in step 1, the solutions established in step 1 are indeed global optima.

Q.E.D.

\section{Proof of Proposition 2}

When $e^{1}$ is observable and contractible, the problem again reduces to a single-dimensional moral hazard problem. Clearly, due to LL, at the optimal solution either $A_{G}$ or $A_{B}$ must be zero. Thus, we consider the following two cases.

\section{Case 1}

In this case, the principal offers a positive wage only when the accounting signal is Good, that is, $A_{G}>A_{B}=0$. For the time being, let's assume $m$ is restricted to be interior, that is, $m \in\left(k_{h}-d_{h}, k_{h}+d_{h}\right)$. In order to motivate the agent to exert $e_{h}^{2}$, the agent's IC constraint must be satisfied, that is,

$$
\frac{k_{h}+d_{h}-m}{2 d_{h}} A_{G}-c^{2} \geq \frac{k_{h}+d_{l}-m}{2 d_{l}} A_{G} \Leftrightarrow\left(d_{l}-d_{h}\right)\left(k_{h}-m\right) A_{G} \geq 2 d_{h} d_{l} c^{2} .
$$

This implies that, in order for the IC to be satisfied, it is necessary that $k_{h}>m$. Thus, the principal solves:

$$
\begin{aligned}
& \max _{A, m, i} E[s-\alpha|i-s|-w a g e]=\max _{A, m} \quad k_{h}-\alpha \frac{\left(m-k_{h}\right)^{2}+d_{h}^{2}}{4 d_{h}}-\frac{k_{h}+d_{h}-m}{2 d_{h}} A_{G} \\
& \text { subject to } A_{G} \geq \frac{2 d_{h} d_{l} c^{2}}{\left(d_{l}-d_{h}\right)\left(k_{h}-m\right)} \text { and } k_{h}>m \\
& \text { (IC }-2) .
\end{aligned}
$$

Clearly, at the optimal solution, $A_{G}=\frac{2 d_{h} d_{l} c^{2}}{\left(d_{l}-d_{h}\right)\left(k_{h}-m\right)}$. Because the objective function is concave in $m$, the first-order condition $m^{*}=k_{h}-\sqrt[3]{\frac{2 d_{h}^{2} d_{l} c^{2}}{\alpha\left(d_{l}-d_{h}\right)}}$ is both necessary and sufficient for optimality. What's more, $m^{*}$ corresponds to a liberal accounting system as $E\left(s-m^{*} \mid\left\{e_{h}^{1}, e_{h}^{2}\right\}\right)=\sqrt[3]{\frac{2 d_{h}^{2} d_{l} c^{2}}{\alpha\left(d_{l}-d_{h}\right)}}>0$. Finally, let's consider choices of $m$ that are not interior, that is, $m \notin\left(k_{h}-d_{h}, k_{h}+d_{h}\right)$. 
In this case, it is easy to show that the principal's optimal $m$ is (infinitely close to) $k_{h}-d_{h}$ and optimal $A_{G}=\frac{2 d_{l} c^{2}}{d_{l}-d_{h}}$. Thus, the optimal $m$ is $\max \left\{k_{h}-\sqrt[3]{\frac{2 d_{h}^{2} d_{l} c^{2}}{\alpha\left(d_{l}-d_{h}\right)}}, k_{h}-d_{h}\right\}$.

\section{Case 2}

In the second case, when the principal offers a positive wage only when the accounting signal is $\mathrm{Bad}$, that is, $A_{B}>A_{G}=0$, following a similar approach as in case 1 , it can be shown the optimal information system is characterized by $m=\min \left\{k_{h}+\sqrt[3]{2 d_{h}^{2} d_{l} c^{2} /\left[\alpha\left(d_{l}-d_{h}\right)\right]}, k_{h}+d_{h}\right\}$, a conservative system, that is, $E\left(s-m^{*} \mid\left\{e_{h}^{1}, e_{h}^{2}\right\}\right)=-\sqrt[3]{\frac{2 d_{h}^{2} d_{l} c^{2}}{\alpha\left(d_{l}-d_{h}\right)}}<0$.

Because the magnitude of biases and expected wage compensation are identical under both cases, the principal receives exactly the same expected payoff.

Q.E.D.

\section{Proof of Proposition 3}

In order to motivate high efforts on both $e^{1}$ and $e^{2}$, multiple IC constraints must be satisfied.

First, the optimal contract must provide incentives to the agent to prefer $\left(e_{h}^{1}, e_{h}^{2}\right)$ to $\left(e_{l}^{1}, e_{h}^{2}\right): A_{G}-A_{B} \geq \frac{2 d_{h} c^{1}}{k_{h}-k_{l}}$, which is the (IC-1) constraint in the proof to Proposition 1. This constraint implies that a the agent must receive a higher reward when the accounting report is Good, that is, $A_{G} \geq A_{B}$.

Second, the optimal contract must also induce the agent to prefer $\left(e_{h}^{1}, e_{h}^{2}\right)$ to $\left(e_{h}^{1}, e_{l}^{2}\right)$, which, for any $m \in\left[k_{h}-d_{h}, k_{h}+d_{h}\right]$, is equivalent to:

$$
\begin{aligned}
\frac{k_{h}+d_{h}-m}{2 d_{h}} A_{G}+\frac{m-\left(k_{h}-d_{h}\right)}{2 d_{h}} A_{B}-c^{2} \geq & \frac{k_{h}+d_{l}-m}{2 d_{l}} A_{G} \\
& +\frac{m-\left(k_{h}-d_{l}\right)}{2 d_{l}} A_{B}
\end{aligned}
$$

Note that (IC-2) $\Leftrightarrow\left(d_{l}-d_{h}\right)\left(k_{h}-m\right)\left(A_{G}-A_{B}\right) \geq 2 d_{h} d_{l} c^{2}$. Because (IC-1) implies $A_{G} \geq A_{B}$, a necessary condition for (IC-2) to be satisfied is $m<k_{h}$ (i.e., a liberal accounting system).

For any $m \notin\left[k_{h}-d_{h}, k_{h}+d_{h}\right]$, the only possibility of having a conservative system is when $m>k_{h}+d_{h}$. In this case, (IC-2) becomes $A_{B}-c^{2} \geq$ $\left[1-\min \left\{\frac{m-\left(k_{h}-d_{l}\right)}{2 d_{l}}, 1\right\}\right] A_{G}+\min \left\{\frac{m-\left(k_{h}-d_{l}\right)}{2 d_{l}}, 1\right\} A_{B}$, or $-c^{2} \geq \max \left\{\frac{k_{h}+d_{l}-m}{2 d_{l}}, 0\right\}$ $\left(A_{G}-A_{B}\right)$, which clearly contradicts $A_{G} \geq A_{B}$.

Q.E.D.

\section{Derivation of Expressions (3)}

The likelihood distribution function, denoted $L_{m}^{2}(z)$, is the probability that $1-\operatorname{Pr}\left(j \mid e_{h}^{1}, e_{l}^{2}\right) / \operatorname{Pr}\left(j \mid e_{h}^{1}, e_{h}^{2}\right) \leq z$, where $j \in\{G, B\}$, conditional on $\left(e_{h}^{1}, e_{h}^{2}\right)$. Note that, when $j=B, 1-\operatorname{Pr}\left(j \mid e_{h}^{1}, e_{l}^{2}\right) / \operatorname{Pr}\left(j \mid e_{h}^{1}, e_{h}^{2}\right)=1-d_{h}\left(m-k_{h}+d_{l}\right) /$ $\left[d_{l}\left(m-k_{h}+d_{h}\right)\right]$, while when $j=G, 1-\operatorname{Pr}\left(j \mid e_{h}^{1}, e_{l}^{2}\right) / \operatorname{Pr}\left(j \mid e_{h}^{1}, e_{h}^{2}\right)=1-d_{h}$ $\left(k_{h}+d_{l}-m\right) /\left[d_{l}\left(k_{h}+d_{h}-m\right)\right]$. It is easy to show that $1-d_{h}$ $\left(k_{h}+d_{l}-m\right) /\left[d_{l}\left(k_{h}+d_{h}-m\right)\right]>1-d_{h}\left(m-k_{h}+d_{l}\right) /\left[d_{l}\left(m-k_{h}+d_{h}\right)\right]$. 
Thus, Expressions (3) are established by noting:

$\forall z<1-d_{h}\left(m-k_{h}+d_{l}\right) /\left[d_{l}\left(m-k_{h}+d_{h}\right)\right]$,

$\operatorname{Pr}\left\{1-\operatorname{Pr}\left(j \mid e_{h}^{1}, e_{l}^{2}\right) / \operatorname{Pr}\left(j \mid e_{h}^{1}, e_{h}^{2}\right) \leq z \mid e_{h}^{1}, e_{h}^{2}\right\}=\operatorname{Pr}\left(j \notin\{G, B\} \mid e_{h}^{1}, e_{h}^{2}\right)=0 ;$

$\forall 1-d_{h}\left(m-k_{h}+d_{l}\right) /\left[d_{l}\left(m-k_{h}+d_{h}\right)\right] \leq z<1-d_{h}\left(k_{h}+d_{l}-m\right) /\left[d_{l}\left(k_{h}+d_{h}-m\right)\right]$, $\operatorname{Pr}\left\{1-\operatorname{Pr}\left(j \mid e_{h}^{1}, e_{l}^{2}\right) / \operatorname{Pr}\left(j \mid e_{h}^{1}, e_{h}^{2}\right) \leq z \mid e_{h}^{1}, e_{h}^{2}\right\}=\operatorname{Pr}\left(j=B \mid e_{h}^{1}, e_{h}^{2}\right)=\left[m-\left(k_{h}-d_{h}\right)\right] /\left(2 d_{h}\right) ;$ and $\forall z \geq 1-d_{h}\left(k_{h}+d_{l}-m\right) /\left[d_{l}\left(k_{h}+d_{h}-m\right)\right]$,

$\operatorname{Pr}\left\{1-\operatorname{Pr}\left(j \mid e_{h}^{1}, e_{l}^{2}\right) / \operatorname{Pr}\left(j \mid e_{h}^{1}, e_{h}^{2}\right) \leq z \mid e_{h}^{1}, e_{h}^{2}\right\}=\operatorname{Pr}\left(j \in\{G, B\} \mid e_{h}^{1}, e_{h}^{2}\right)=1$. Q.E.D.

\section{Proof of Proposition 4}

We proceed in two steps. First, we demonstrate the proposition holds for a restricted program in which the information system cutoff $m$ is restricted to be $m \in\left[k_{h}-d_{h}, k_{h}\right)$. Second, we show the solution to the restricted program is also optimal when $m$ is unrestricted.

Step 1. When $m$ is restricted to $m \in\left[k_{h}-d_{h}, k_{h}\right)$.

Notice that, depending on $m$, only one of the IC constraints binds. Specifically, define:

$m_{1} \equiv k_{l}-\frac{c^{2} d_{l}\left(k_{h}-k_{l}\right)}{c^{1}\left(d_{l}-d_{h}\right)}<m_{2} \equiv k_{h}-\frac{c^{2} d_{h}\left(k_{h}-k_{l}\right)}{c^{1}\left(d_{l}-d_{h}\right)}$.

It can be verified that, for $\forall m<m_{1}$, (IC-1) is the binding constraint; for $\forall m \in\left[m_{1}, m_{2}\right),(\mathrm{IC}-3)$ is the binding constraint; and for $\forall m \in\left[m_{2}, k_{h}\right)$, (IC-2) is the binding constraint.

We start by noting that the optimal solution $m$ cannot be smaller than $m_{1}$, that is, (IC-1) cannot be the only binding constraint. This is because when $m<m_{1}$ the expected wage payment is decreasing in $m$ (i.e., $\left.\frac{\partial\left[\frac{k_{h}+d_{h}-m}{2 d_{h}}\right.}{\partial m} \frac{2 d_{h} c^{1}}{k_{h}-k_{l}}\right]=0$ ) and the expected loss from decision $i, \alpha \frac{\left(m-k_{h}\right)^{2}+d_{h}^{2}}{4 d_{h}}$, is also decreasing in $m$ as $m<m_{1}<k_{h}$; and the principal is strictly better off to increase $m$. Thus, the optimal $m$ has to lie in $\left[m_{1}, k_{h}\right)$. Because for now we focus on interior $m$, therefore the optimal $m$ is in the interval of [ $\left.\max \left\{m_{1}, k_{h}-d_{h}\right\}, k_{h}\right)$.

Furthermore, it is straightforward to show that at the optimal solution $A_{G}>A_{B}=0$.

We will focus on the case where (IC-2) binds. This is because our paper is mainly about motivating effort that reduces uncertainty to assist decision making and (IC-2) highlights the situation where the decision-facilitating effort constitutes the main obstacle in motivating the agent. ${ }^{15}$ With (IC-2) as the binding constraint, it is straightforward to show by the first-order condition that the optimal

15. Intuitively, the case where (IC-3) binds relates to situations that relative to the meanincreasing effort, the cost of uncertainty-reducing effort is not very high hence not the main obstacle in incentivizing the agent. 


$$
\begin{aligned}
& \widetilde{m}=k_{h}-\sqrt[3]{\frac{2 d_{h}^{2} d_{l} c^{2}}{\alpha\left(d_{l}-d_{h}\right)}} \\
& A_{G}=\frac{2 d_{h} d_{l} c^{2}}{\left(d_{l}-d_{h}\right)\left(k_{h}-\widetilde{m}\right)}, A_{B}=0 .
\end{aligned}
$$

To guarantee that this is indeed an interior optimal solution, we need the following conditions to hold:

(1) $\widetilde{m} \geq k_{h}-d_{h}$, and

(2) $\widetilde{m}, A_{G}$, and $A_{B}$ satisfy the (IC-3) constraint.

Condition 1 ensures that $\widetilde{m}$ is indeed interior. Condition 2 ensures that (IC-2) is the pressing constraint. Because (IC-2) is the pressing constraint if and only if $\widetilde{m} \geq m_{2}$, condition 2 is equivalent to verifying that $\widetilde{m} \geq m_{2}$. To establish the parameter region over which conditions 1 and 2 hold, we consider two cases depending on whether $m_{2} \leq k_{h}-d_{h}$ or $m_{2}>k_{h}-d_{h}$. It is easy to verify that $m_{2} \leq k_{h}-d_{h} \Leftrightarrow c^{2} \geq \hat{c}^{2} \equiv \frac{\left(d_{l}-d_{h}\right) c^{1}}{k_{h}-k_{l}}$.

Case 1: $m_{2} \leq k_{h}-d_{h} \Leftrightarrow c^{2} \geq \widehat{c^{2}} \equiv \frac{\left(d_{l}-d_{h}\right) c^{1}}{k_{h}-k_{l}}$. This means that condition 1 implies condition 2 and we only need to check condition 1, which requires $\widetilde{m} \geq k_{h}-d_{h} \Leftrightarrow c^{2} \leq \overline{\overline{c^{2}}} \equiv \frac{\alpha d_{h}\left(d_{l}-d_{h}\right)}{2 d_{l}}$.

For $c^{2} \in\left[\widehat{c}^{2}, \underline{\underline{c^{2}}}\right]$ to be non-empty, we note that a sufficient and necessary condition is $\alpha \geq \underline{\underline{\alpha}} \equiv \frac{2 d_{l} c^{1}}{\left(k_{h}-k_{l}\right) d_{h}}$.

Case 2: $m_{2}>k_{h}-d_{h} \Leftrightarrow c^{2}<\widehat{c^{2}}$. This means that condition 2 implies condition 1 and we only need to check condition 2 , which requires

$$
\widetilde{m} \geq m_{2} \Leftrightarrow c^{2} \geq \underline{c}^{2} \equiv \frac{c^{1}\left(d_{l}-d_{h}\right)}{k_{h}-k_{l}} \sqrt{\frac{2 c^{1} d_{l}}{\alpha\left(k_{h}-k_{l}\right) d_{h}}} .
$$

It is easy to verify that $\left[\underline{c}^{2}, \widehat{c^{2}}\right)$ is not an empty set iff $\alpha>\underline{\alpha}$.

Lastly, we need to consider the corner solution when $c^{2}>\underline{\underline{c^{2}}}$. Note when $\alpha>\underline{\alpha}, c^{2}>\overline{\overline{c^{2}}} \Rightarrow m_{2}<k_{h}-d_{h}$, which implies (IC-2) is the only binding constraint for all interior $m$ 's. However, when $c^{2}>\underline{\underline{c}}^{2}$ the solution given by the FOC $\widetilde{m}$ falls outside of the boundary, that is, $\widetilde{m}<k_{h}-d_{h}$, which means the optimal $m$ is the corner solution of $k_{h}-d_{h}$ with $\left\{A_{B}=0, A_{G}=\frac{2 d_{l} c^{2}}{d_{l}-d_{h}}\right\} .{ }^{16}$

Step 2. Optimal solution when $m$ is restricted to be $m \notin\left[k_{h}-d_{h}, k_{h}\right)$.

Given Proposition 2, the principal's optimal $m$ must be liberal. Thus, the only possibility here is $m<k_{h}-d_{h}$. Clearly, the optimal $m$ is (infinitely

16. The optimal solution when $c^{2}<\underline{c}^{2}$ is fairly complicated and involves (IC-3) as the binding constraint. For completeness, we lay out the solution below (details are available upon request): for $\forall c^{2} \in\left[\underline{\underline{c}}^{2}, \underline{c}^{2}\right)$, the optimal contract is $\left\{A_{B}=0, A_{G}=\frac{2\left(c^{1}+c^{2}\right) d_{h} d_{l}}{\left(k_{h}-m\right) d_{l}-\left(k_{l}-m\right) d_{h}}\right\}$ and $m=m_{2} ; \forall c^{2}<\underline{\underline{c^{2}}}$, the optimal contract is $\left\{A_{B}=0, A_{G}=\frac{2\left(c^{1}+c^{2}\right) d_{h} d_{l}-}{\left(k_{h}-m\right) d_{l}-\left(k_{l}-m\right) d_{h}}\right\}$ and $m=\min \left\{\max \left\{k_{h}-d_{h}, m_{1}, \underset{t}{\arg \max }\left\{k_{h}-\alpha \frac{\left(t-k_{h}\right)^{2}+d_{h}^{2}}{4 d_{h}}-\frac{\left(k_{h}+d_{h}-t\right)\left(c^{1}+c^{2}\right) d_{l}}{\left(k_{h}-t\right) d_{l}-\left(k_{l}-t\right) d_{h}}\right\}\right\}, k_{h}\right\}$. 
close to) $k_{h}-d_{h}$ because any lower $m$ only increases expected wage compensation and doesn't improve decision making. This implies the corner solution of $m=k_{h}-d_{h}$ weakly dominates all $m<k_{h}-d_{h}$. Because the principal's payoff function is continuous in $m$ and the boundary solution of $m=k_{h}-d_{h}$ has been considered above in step 1, the solutions established in step 1 are indeed global optima.

Thus, cases 1 and 2 together with the corner solution $m=k_{h}-d_{h}$ above prove part (i) of Proposition 4. The comparative statics are immediate from the steps above, and, hence omitted.

Q.E.D.

\section{Proof of Proposition 5}

The strategy of this proof is to derive and compare the principal's expected payoff from motivating each of the four effort combinations: $\left(e_{h}^{1}, e_{h}^{2}\right)$, $\left(e_{l}^{1}, e_{h}^{2}\right),\left(e_{h}^{1}, e_{l}^{2}\right)$, and $\left(e_{l}^{1}, e_{l}^{2}\right)$.

(1) Principal's payoff from motivating $\left(e_{h}^{1}, e_{h}^{2}\right)$.

From Proposition 4, we have for $\forall c^{2}>\underline{c^{2}}$, the optimal contract is $\left\{A_{B}=0, A_{G}=\frac{2 d_{l} c^{2}}{d_{l}-d_{h}}, m=k_{h}-d_{h}\right\}$; substituting these expressions into the principal's objective function obtains the principal's expected payoff as $P\left(e_{h}^{1}, e_{h}^{2}\right)=k_{h}-\alpha \frac{d_{h}}{2}-\frac{2 d_{1} c^{2}}{d_{l}-d_{h}} ;$ for $\forall c^{2} \in\left[\underline{c}^{2}, \overline{\overline{c^{2}}}\right]$ the optimal contract is $\left\{A_{B}=0, A_{G}=\frac{2 d_{h} d_{l} c^{2}}{\left(d_{l}-d_{h}\right)\left(k_{h}-m\right)}, m=k_{h}-\sqrt[3]{\frac{2 d_{h}^{2} d_{l} c^{2}}{\alpha\left(d_{l}-d_{h}\right)}}\right.$, under which the principal's expected payoff is:

$$
P\left(e_{h}^{1}, e_{h}^{2}\right)=k_{h}-\alpha \frac{\left[\frac{2 d_{h}^{2} d_{l} c^{2}}{\alpha\left(d_{l}-d_{h}\right)}\right]^{\frac{2}{3}}+d_{h}^{2}}{4 d_{h}}-\frac{\left(\sqrt[3]{\frac{2 d_{h}^{2} d_{l} c^{2}}{\alpha\left(d_{l}-d_{h}\right)}}+d_{h}\right) 2 d_{h} d_{l} c^{2}}{2 d_{h}\left(\left(d_{l}-d_{h}\right) \sqrt[3]{\frac{2 d_{h}^{2} d_{l} c^{2}}{\alpha\left(d_{l}-d_{h}\right)}}\right.} .
$$

For $c^{2}<\underline{c}^{2}$, note when $c^{2}$ is small (say, close to 0 ), $\underline{c}^{2}$ is also small (i.e., $\lim _{c^{1} \rightarrow 0} \underline{c}^{2}=0$ ). In other words, when $c^{1}$ is sufficiently small and $c^{2}<\underline{c}^{2}$, the incentive problem is close to nonexistent. Thus, the principal's expected payoff is close to first best: $\forall c^{2}<\underline{c}^{2}$ and $c^{1}$ small, $P\left(e_{h}^{1}, e_{h}^{2}\right) \approx k_{h}-\alpha \frac{d_{h}}{4}$.

(2) Principal's payoff from motivating $\left(e_{l}^{1}, e_{h}^{2}\right)$.

The problem reduces to a single-dimensional moral hazard problem where the incentive problem is only present for $e^{2}$ and all distributions have a mean $k_{l}$. In this case, an upper bound on the principal's expected payoff is obtained from a relaxed program where the only incentive constraint stipulates that $\left(e_{l}^{1}, e_{h}^{2}\right)$ is preferred to $\left(e_{l}^{1}, e_{l}^{2}\right)$. When $c^{2}>\underline{\underline{c^{2}}}$, an optimal information system is a corner solution at $m=k_{l}-d_{h}$, and the principal's expected payoff is $P\left(e_{l}^{1}, e_{h}^{2}\right)=k_{l}-\alpha \frac{d_{h}}{2}-\frac{2 d_{l} c^{2}}{d_{l}-d_{h}}$. When $c^{2}<\underline{\underline{c^{2}}}$, an optimal information system is $m=k_{l}-\sqrt[3]{\frac{2 d_{h}^{2} d_{l} c^{2}}{\alpha\left(d_{l}-d_{h}\right)}}$, and the principal's expected payoff is: 


$$
P\left(e_{l}^{1}, e_{h}^{2}\right)=k_{l}-\alpha \frac{\left[\frac{2 d_{h}^{2} d_{l} c^{2}}{\alpha\left(d_{l}-d_{h}\right.}\right)^{\frac{2}{3}}+d_{h}^{2}}{4 d_{h}}-\frac{\left(\sqrt[3]{\frac{2 d_{h}^{2} d_{l} c^{2}}{\alpha\left(d_{l}-d_{h}\right)}}+d_{h}\right) 2 d_{h} d_{l} c^{2}}{2 d_{h}\left(\left(d_{l}-d_{h}\right) \sqrt[3]{\frac{2 d_{h}^{2} d_{l} c^{2}}{\alpha\left(d_{l}-d_{h}\right)}}\right)} .
$$

(3) Principal's payoff from motivating $\left(e_{h}^{1}, e_{l}^{2}\right)$.

The problem reduces to a single-dimensional moral hazard problem where the incentive problem is only present for $e^{1}$ and all distributions have $2 d_{l}$ as their support. Thus, by a slight adaptation of Proposition 1 , the optimal information system is a conservative one with $m=k_{h}+\frac{2 d_{l} c^{1}}{\alpha\left(k_{h}-k_{l}\right)}$, when $c^{1}$ is small. In this case, the principal's expected payoff is

$$
P\left(e_{h}^{1}, e_{l}^{2}\right)=k_{h}-\alpha \frac{\left(\frac{2 d_{l} c^{1}}{\alpha\left(k_{h}-k_{l}\right)}\right)^{2}+d_{l}^{2}}{4 d_{l}}-\frac{d_{l}-\frac{2 d_{l} l^{1}}{\alpha\left(k_{h}-k_{l}\right)}}{2 d_{l}} \frac{2 d_{l} c^{1}}{k_{h}-k_{l}} .
$$

(4) Principal's payoff from motivating $\left(e_{l}^{1}, e_{l}^{2}\right)$.

In this case, the principal would optimally forgo all contingent payments and by Lemma 1 set $m=k_{1}$. The principal's expected payoff is thus $P\left(e_{l}^{1}, e_{l}^{2}\right)=k_{l}-\alpha \frac{d_{l}}{4}$.

Now, we compare the principal's payoff from the four effort combinations when $c^{1}$ is sufficiently small.

(i) Comparing $P\left(e_{h}^{1}, e_{h}^{2}\right)$ with $P\left(e_{l}^{1}, e_{h}^{2}\right)$.

$\forall c^{2} \geq \underline{c}^{2}, P\left(e_{h}^{1}, e_{h}^{2}\right)-P\left(e_{l}^{1}, e_{h}^{2}\right)=k_{h}-k_{l}>0$; and $\forall c^{2}<\underline{c}^{2}, P\left(e_{h}^{1}, e_{h}^{2}\right)-P\left(e_{l}^{1}, e_{h}^{2}\right) \approx k_{h}-k_{l}>0$. Thus, $P\left(e_{h}^{1}, e_{h}^{2}\right)>P\left(e_{l}^{1}, e_{h}^{2}\right)$.

(ii) Comparing $P\left(e_{h}^{1}, e_{l}^{2}\right)$ with $P\left(e_{l}^{1}, e_{l}^{2}\right)$.

With $c^{1}$ sufficiently small, $P\left(e_{h}^{1}, e_{l}^{2}\right)>P\left(e_{l}^{1}, e_{l}^{2}\right)$.

(iii) Comparing $P\left(e_{h}^{1}, e_{h}^{2}\right)$ with $P\left(e_{h}^{1}, e_{l}^{2}\right)$.

When $c^{1}$ is small, $P\left(e_{h}^{1}, e_{l}^{2}\right)$ is close to $k_{h}-\alpha \frac{d_{l}}{4}$ and $P\left(e_{h}^{1}, e_{h}^{2}\right)$ evaluated at $c^{2}=0$ is in the neighborhood of $k_{h}-\alpha \frac{d_{h}}{4}$. Hence, $P\left(e_{h}^{1}, e_{h}^{2}\right)>P\left(e_{h}^{1}, e_{l}^{2}\right)$, when $c^{2}=0$. Finally, observe that $P\left(e_{h}^{1}, e_{h}^{2}\right)$ is strictly decreasing in $c^{2}$ without bound, while $P\left(e_{h}^{1}, e_{l}^{2}\right)$ is independent of $c^{2}$. Hence, $P\left(e_{h}^{1}, e_{l}^{2}\right)>P\left(e_{h}^{1}, e_{h}^{2}\right)$ if and only if $c^{2}$ is above a certain threshold $\bar{c}^{2}$.

Q.E.D.

\section{References}

Arya, A., J. Glover, and K. Sivaramakrishnan. 1997. The interaction between decision and control problems and the value of information. The Accounting Review 72 (4): 561-74.

Bagnoli, M., and S. Watts. 2005. Conservative accounting choices. Management Science 51 (5): 786-801.

Blackwell, D. 1951. Comparison of experiments. Proceedings of the Second Berkeley Symposium on Mathematics, Statistics and Probability, 93-102.

Bowen, R. M., S. Rajgopal, and M. Venkatachalam. 2010. Accounting discretion, corporate governance and firm performance. Contemporary Accounting Research 25 (2): 351-405. 
Chen, Q., T. Hemmer, and Y. Zhang. 2007. On the relation between conservatism in accounting standards and earnings management. Journal of Accounting Research 45 (3): 541-66.

Christensen, J., and J. Demski. 2003. Accounting theory: An information content perspective. New York: Irwin/McGraw-Hill.

Demski, J. 1980. Information analysis, 2nd ed. London: Addison Wesley.

Demski, J., and R. Dye. 1999. Risk, return, and moral hazard. Journal of Accounting Research 37 (1): 27-55.

Dye, R. 1985. Strategic accounting choice and the effects of alternative financial reporting requirements. Journal of Accounting Research 23 (2): 544-74.

Dye, R. 2002. Classifications manipulation and Nash accounting standards. Journal of Accounting Research 40 (4): 1125-62.

Fields, T., T. Lys, and L. Vincent. 2001. Empirical research on accounting choice. Journal of Accounting and Economics 31 (1): 255-307.

Francis, J., R. LaFond, P. Olsson, and K. Schipper. 2005. The market pricing of accruals quality. Journal of Accounting and Economics 39 (2): 295-327.

Gigler, F., and T. Hemmer. 2001. Conservatism, optimal disclosure policy, and the timeliness of financial reports. The Accounting Review 76 (4): 471-93.

Gjesdal, F. 1982. Information and incentives: The agency information problem. Review of Economic Studies 49 (3): 373-90.

Grossman, S., and O. Hart. 1983. An analysis of the principal-agent problem. Econometrica 51 (1): 7-45.

Healy, P. M., and K. G. Palepu. 1996. Using capital structure to communicate with investors: The case of CUC International. Journal of Applied Corporate Finance 8 (4): $30-44$.

Healy, P. M., and J. Wahlen. 1999. A review of the earnings management literature and its implications for standard setting. Accounting Horizons 13 (4): $365-83$.

Hirshleifer, D., and Y. Suh. 1992. Risk, managerial effort, and project choice. Journal of Financial Intermediation 2 (3): 308-45.

Holmstrom, B., and P. Milgrom. 1987. Aggregation and linearity in the provision of intertemporal incentives. Econometrica 55 (2): 303-28.

Ijiri, Y. 1975. Theory of accounting measurement. Sarasota, FL: American Accounting Association.

Kim, S. 1995. Efficiency of an information system in an agency model. Econometrica 63 (1): 89-102.

Kwon, Y. 2005. Accounting conservatism and managerial incentives. Management Science 51 (11): 1626-32.

Kwon, Y., P. Newman, and Y Suh. 2001. The demand for accounting conservatism for management control. Review of Accounting Studies 6 (1): 29-51.

Meth, B. 1996. Reduction of outcome variance: Optimality and incentives. Contemporary Accounting Research 13 (1): 309-28.

Subramanyam, K. R. 1996. The pricing of discretionary accruals. Journal of Accounting and Economics 22: 249-81. 
Sung, J. 1995. Linearity with project selection and controllable diffusion rate in continuous-time principal-agent problems. RAND Journal of Economics 26 (4): $720-43$.

Venugopalan, R. 2001. Conservatism in accounting: Good or bad? Working paper, University of Minnesota.

Watts, R., and J. Zimmerman. 1986. Positive accounting theory. Englewood Cliffs, NJ: Prentice-Hall. 